\title{
Anisotropies in the Distribution of Satellite Galaxies
}

\author{
Dennis Zaritsky \\ UCO/Lick Observatories and Board of Astronomy and Astrophysics, \\ Univ. of California at Santa Cruz, Santa Cruz, CA, 95064 \\ Rodney Smith \\ Department of Physics, P.O. Box 913, University of Wales, College of Cardiff, \\ Cardiff CF2 3YB, Wales. \\ Carlos S. Frenk \\ Department of Physics, South Road, University of Durham, Durham, DH1 3LE, England \\ and \\ Simon D.M. White \\ Max-Planck-Institut für Astrophysik, Karl-Schwarzschild-Strasse 1, \\ D-85748 Garching bei München, Germany
}

Received __; accepted _ 


\begin{abstract}
We find that satellites of isolated disk galaxies at projected radii between 300 and $500 \mathrm{kpc}$ are distributed asymmetrically about the parent galaxy and aligned preferentially with the disk minor axis. The dynamical timescale at these radii is sufficiently long that the shape of this distribution must reflect the formation history of the outer halo rather than its internal evolution. We also find that the orbital angular momenta of satellites at projected major axis distances of $\lesssim$ $200 \mathrm{kpc}$ tend to align with that of the central disk. These results demonstrate that satellites are dynamically related to their primary galaxy. Satellites drawn from current simulations of hierarchical galaxy formation exhibit neither the systematic alignment nor the net rotation with the central disk that we find in the data.
\end{abstract}




\section{Introduction}

Although it has become increasingly evident over the last two decades that isolated galaxies are surrounded by large and massive unseen halos (Faber \& Gallagher 1979; Zaritsky \& White 1994), the mass distribution in these halos remains weakly constrained. Dark matter is generally thought to constitute between 90 and $99.5 \%$ of the mass in the universe and a thorough understanding of its distribution is critical to the study of galaxy evolution and cosmology. The assumption that the halos of galaxies are spherical at large radii is often made, but has little observational or theoretical support. Satellite galaxies provide the best available test of this assumption.

Null or marginal detections of anisotropy in the relative orientation of galaxies (Hawley \& Peebles 1975; Sharp et al. 1979; MacGillivray et al. 1982) have mostly suggested that galaxies are distributed uniformly in angle about each other. A notable exception is the study of Holmberg (1969) which found that satellite galaxies at projected radii $<50 \mathrm{kpc}$ tend to congregate near the poles of their spiral primaries. Because this anisotropy was seen at small projected radii, it has most often been interpreted as the result of selection effects or dynamical evolution. However, neither of these alternatives appears able to account for the magnitude of the effect (Quinn \& Goodman 1986). In this Letter, we present evidence for an anisotropic distribution of satellite galaxies that is similar to that seen by Holmberg, but which exists at much larger radii, where neither selection biases nor dynamical evolution seem a plausible explanation. 


\section{Data and Analysis}

\subsection{The Spatial Distribution of Satellite Galaxies}

Our data consist of (1) projected positions and radial velocities for 115 satellite galaxies of 69 nearby (distance $<100 \mathrm{Mpc}$, for $\mathrm{H}_{0}=75 \mathrm{~km} \mathrm{~s}^{-1}$ $\mathrm{Mpc}^{-1}$ as assumed throughout) isolated disk galaxies (the primaries) similar in luminosity to our own Galaxy, and (2) a measurement of the rotation sense of the disk for 57 of these primaries. The selection of primary galaxies, the identification of satellites, and all complementary observations are discussed elsewhere (Zaritsky et al. 1993; Zaritsky et al. 1996). Satellites are defined to have projected separations from their primaries that are less than $500 \mathrm{kpc}$ and velocities relative to their primaries that are less than $500 \mathrm{~km} \mathrm{~s}^{-1}$. In Figure 1, we show the satellite distribution projected on the sky and oriented so that each primary is located at the origin with its major axis placed along the horizontal axis.

The elongation of the satellite distribution along the disk minor axis is evident both in the full dataset (left panel) and for the 72 satellites of the 48 primaries that are inclined at least $45^{\circ}$ to the line-of-sight (right panel). Observational biases due either to the telescope or to the instruments cannot be responsible for this anisotropy because the position angles of the primaries are randomly distributed on the sky and were not taken into account when setting up the observations. Furthermore, our data come from a variety of telescopes and instruments, as well as from the literature. The apparent anisotropy is qualitatively similar to that found by Holmberg (1969), but is occurring at projected radii much larger than the $50 \mathrm{kpc}$ limit of his dataset. We find no evidence for a polar alignment of satellites at projected radii $<200 \mathrm{kpc}$, and the 
visual impression is that the satellite distribution is flattened in the opposite sense along the disk major axis at these radii. This apparent flattening is not statistically significant. Finally, we cannot test Holmberg's result because we have only 9 satellites at $r_{p}<50 \mathrm{kpc}$

We estimate the statistical significance of the polar alignment at large projected radii by applying a Kolmogorov-Smirnoff (K-S) statistic to compare the distribution of position angles relative to the disk major axis (reduced to the range 0 to $90^{\circ}$ ) with the uniform distribution expected for circular symmetry. We begin with the twenty satellites at the largest projected separations. We apply the K-S test. We then add satellites one at a time, stepping radially inward, and reapplying the K-S test to each enlarged subsample. The results as a function of the lower limit in projected radius are shown in Figure 2 both for the full sample of 115 satellites (solid line) and for a restricted sample of 72 satellites, for which the primary is inclined by at least $45^{\circ}$ to the line-of-sight (dashed line). In both cases, the anisotropy is significant with greater than $99 \%$ confidence for projected separations $\gtrsim 250 \mathrm{kpc}$, and is absent for $r_{p} \lesssim 200 \mathrm{kpc}$. Note that about a third of our sample lies beyond $250 \mathrm{kpc}$, and about half lies within $200 \mathrm{kpc}$. The apparent axial ratio of the satellite distribution in the outer regions is roughly 5:3. A polar alignment of satellites is also observed among the satellites of our own Galaxy (Hartwick 1996), although at a much lower significance level.

These K-S confidence levels assume that each satellite is independently drawn from the parent distribution. We now test whether our conclusions are affected by spatial correlations among satellites. Such correlations could arise because interlopers (i.e., galaxies projected along the line of sight, but not physically associated with the primary) are clustered. We proceed by randomly 
rejecting all but one satellite at $r_{p}>250 \mathrm{kpc}$ for each primary-satellite system and then repeating the analysis. The results are shown in Figure 2 as a dotted line and are fully consistent with those found for the original sample. We conclude that the anisotropy is real.

\subsection{The Net Rotation of the Satellite Population}

Our data enable us to determine whether the satellite ensemble rotates with the central galaxy disk. Using the disk major axis and rotation direction to align the 57 systems for which rotation has been measured, we estimate the ensemble mean rotation by averaging the line-of-sight velocity difference between satellite and primary using opposite signs on the two sides of the projected rotation axis. We find a net rotation velocity of $34 \pm 14 \mathrm{~km} \mathrm{~s}^{-1}$ in the same sense as the disk; the data are shown in Figure 3. The true mean rotation velocity of the satellite ensemble is presumably larger than this because the primary disks are observed at a range of inclinations. Correcting, using the mean inclination of the primary disks $\left(54^{\circ}\right)$, gives an "edge-on" mean rotation velocity of $v_{R O T}=42 \mathrm{~km} \mathrm{~s}^{-1}$.

Although the satellite population rotates systematically, the dispersion about the mean motion is large. Adopting a flat "rotation curve" we measure a dispersion of $136 \mathrm{~km} \mathrm{~s}^{-1}$, implying $v_{R O T} / \sigma=0.31$. This ratio is similar both to that observed in giant elliptical galaxies (cf. van der Marel 1991), and to that

measured for dark halos in N-body simulations (Frenk et al. 1988, Cole \& Lacey 1996). It is, however, quite unusual, either for galaxies or for simulated halos, to find such values when rotation is about the minor axis. In N-body simulations there is generally a poor correlation between the angular momenta of the inner and outer regions of a halo. 


\section{Discussion}

Three possibilities suggest themselves for connecting the observed satellites to the underlying dark matter: (1) the satellites trace the dark matter, that is their spatial and kinematic distributions can be considered a Poisson sampling from the corresponding dark matter distributions; (2) the satellite and dark matter distributions differ because the satellite population has evolved dynamically from its initial state (e.g., dynamical friction has preferentially removed satellites on prograde or plunging orbits); (3) the satellites do not trace the dark matter because they formed in a biased way relative to the dark matter. There are at least two arguments against the second option. The orbital timescale for distant satellites is a large fraction of the age of the universe so that such strong dynamical separation seems unlikely. In addition, processes like tidal disruption and dynamical friction should be stronger at small radii where the dynamical times are shorter, whereas we see anisotropy only at $r_{p}>250$ kpc. The third option, biased galaxy formation, may indeed lead to differences between the distributions of satellites and of dark matter, but current models give no reason to suspect that the elongation of the satellite system should be perpendicular to that of the dark matter. Bias appears to enhance the contrast of the filaments and sheets already present in the dark matter distribution (e.g., White et al. 1987). In effect, we are therefore left with the first option, that the elongation of the outer satellite distribution reflects that of the mass. The difficulty here is to understand why the outer halo should be systematically elongated perpendicular to the central disk.

The anisotropy we have detected would seem most consistent with a near prolate distribution of satellites at large radii. The apparent axial ratio of the satellite distribution is quite consistent with the typical values found for dark 
matter halos in cosmological simulations, where 1:0.8:0.65 are representative values near the halo virial radius (corresponding to $\sim 180 \mathrm{kpc}$ for our galaxies) and more extreme values are common at larger radii (Efstathiou et al. 1988; Frenk et al. 1988; Cole \& Lacey 1996). Note, however, that most studies have found that in the standard hierarchical model the inner and outer regions of halos are only weakly related; furthermore, rotation, when significant, is almost always around one of the shortest axes (e.g., Barnes \& Efstathiou 1987; Frenk et al. 1988; Warren et al. 1992). The rotation sense of our satellite population and the orientation of the central galaxy are thus both puzzling in the context of these models.

To explore this further, we constructed a sample of simulated satellite galaxies drawn from the simulations of Navarro, Frenk, \& White $(1994,1995)$. The sample was chosen from simulations of the evolution of isolated disk galaxies using the selection criteria of our observational dataset. The thirty simulations, each typically containing 1 to 2 satellites, are viewed from random angles in order to produce pseudo-observational samples of 95 satellites (corresponding to our sample of real satellites of primaries with known rotation sense). None of the five samples we constructed showed any sign of a significant deviation from circular symmetry when the "observations" were superposed and aligned in the same manner as the real data. For these simulations, at least, the correlation between galaxy angular momentum and satellite position is too weak to reproduce our observational result. It is important to note that, as expected, the outer halos in these simulations have elongated shapes which align with nearby structures. The problem is that the orientation of the central disk appears uncorrelated with these preferred directions.

We also examine whether the simulations produce a coherent rotation of 
the satellite ensemble when they are superposed in the same way as the real data. We calculate the correlation between major axis projected distance and satellite-primary velocity difference for our five artificial samples, but in no case do we find a significant correlation or an apparent rotation exceeding $10 \mathrm{~km} \mathrm{~s}^{-1}$. In contrast, the correlation for the real data, shown in Figure 3, is significant at the $99.4 \%$ level (Spearman rank coefficient $=0.349$ ) while the net rotation is $34 \mathrm{~km} \mathrm{~s}^{-1}$. Once again the angular momentum of the central galaxies in the simulations appears to be more weakly related to the properties of the satellite population than is the case in the real data.

Navarro et al. (1995) already noted that angular momentum losses during formation of these simulated disks were too great for the disks to be compatible with observed spirals. They suggested a possible resolution of this problem - that feedback from early star formation might keep much of the gas hot, allowing it to settle later and with less angular momentum loss into the disk. If this indeed happened, the properties of the outer halo might plausibly be more faithfully reflected in the disk than they are in the simulations. The correlation between the angular momentum of the disk and that of the satellite population might then be understood. The apparent elongation along the rotation axis would, however, remain a puzzle.

In summary, we find that the satellite distribution in the outer halo regions of isolated spiral galaxies is elongated in the direction perpendicular to the stellar disk. In addition the satellite population tends to rotate in the same sense as the central disk even though its typical physical scale is much larger (200 kpc as opposed to $5 \mathrm{kpc}$ ). The rotation corresponds to $v / \sigma \sim 0.3$ for the satellite system when the central disks are seen edge-on. Current simulations of galaxy formation do not reproduce these regularities. There are still some 
missing pieces in our understanding of the galaxy formation process.

ACKNOWLEDGMENTS: DZ acknowledges partial financial support from NASA through HF-1027.01-91A, a grant from the California Space Institute, and HST grant AR-06370.01-95A. CSF acknowledges receipt of a PPARC Senior Research Fellowship. 


\section{References}

Barnes, J., \& Efstathiou, G. 1987, ApJ, 319, 575

Cole, S., \& Lacey, C. 1996, MNRAS, 281, 716

Efstathiou, G., Frenk, C.S., White, S.D.M., \& Davis, M. 1988, MNRAS, 235, 715

Frenk, C.S., White, S.D.M., Efstathiou, G. \& Davis, M. 1988, ApJ, 327, 507

Faber, S.M., \& Gallagher, J.S. 1979, ARA \& A, 2, 135

Hartwick, F.D.A. 1996 in Formation of the Galactic Halo... Inside and Out, ASP Conf. Series vol 92, eds. H. Morrison \& A. Sarajedini, 444

Holmberg, E. 1969, Ark. Astron., 5, 305

Navarro, J.F., Frenk, C.S., \& White, S.D.M. 1994, MNRAS, 267, L1

Navarro, J.F., Frenk, C.S., \& White, S.D.M. 1995, MNRAS, 275, 56

Quinn, P.J. \& Goodman, J. 1986, ApJ, 309, 472

Sharp, N.A., Lin, D.N.C., \& White, S.D.M. 1979, MNRAS, 187, 287

van den Marel, R.P. 1991, MNRAS, 253, 710

Warren, M.S., Quinn, P.J., Salmon, J.K., \& Zurek, W.H. 1992, ApJ, 399, 405

White, S.D.M., Frenk, C.S., Davis, M., \& Efstathiou, G. 1987, ApJ, 313, 505

Zaritsky, D., Smith, R., Frenk, C., \& White, S.D.M. 1993, ApJ, 405, 464

Zaritsky, D., Smith, R., Frenk, C., \& White, S.D.M. 1996, ApJ, in press.

Zaritsky, D., \& White, S.D.M. 1994, ApJ, 435, 599. 


\section{Figure Captions}

1) The projected distribution of satellite galaxies referenced to a primary galaxy centered on the origin with its major axis aligned with the horizontal. The left panel includes all 115 satellites. The right panel includes the 72 satellites of the 48 primaries that are inclined more than $45^{\circ}$ with respect to the line-of- sight.

2) The results of a K-S test comparison between the observed and a uniform azimuthal distribution of satellites. The sample lower boundary is set in projected separation and is plotted along the $\mathrm{x}$ axis. Probability refers to the probability that the observed azimuthal distribution is not drawn from a uniform distribution. The solid line represents the full sample of 115 satellites, the dashed line represents the sample of 72 satellites for which the the primary disk is inclined at least $45^{\circ}$ to the line-of-sight, and the dotted line represents the sample where multiple satellite groups have been removed (see text).

3) The rotation curve of the satellite system along the disk major axis. The lower panel includes the data for the 61 satellites of primaries inclined more than $45^{\circ}$ to the line-of-sight for which we have a disk rotation measurement. Each system is oriented so that the primary disk at positive radius has positive velocity. The upper panel shows a running mean (dashed line) and median (solid line) of the nearest nine satellite galaxy velocities. 
Fig. 1.-

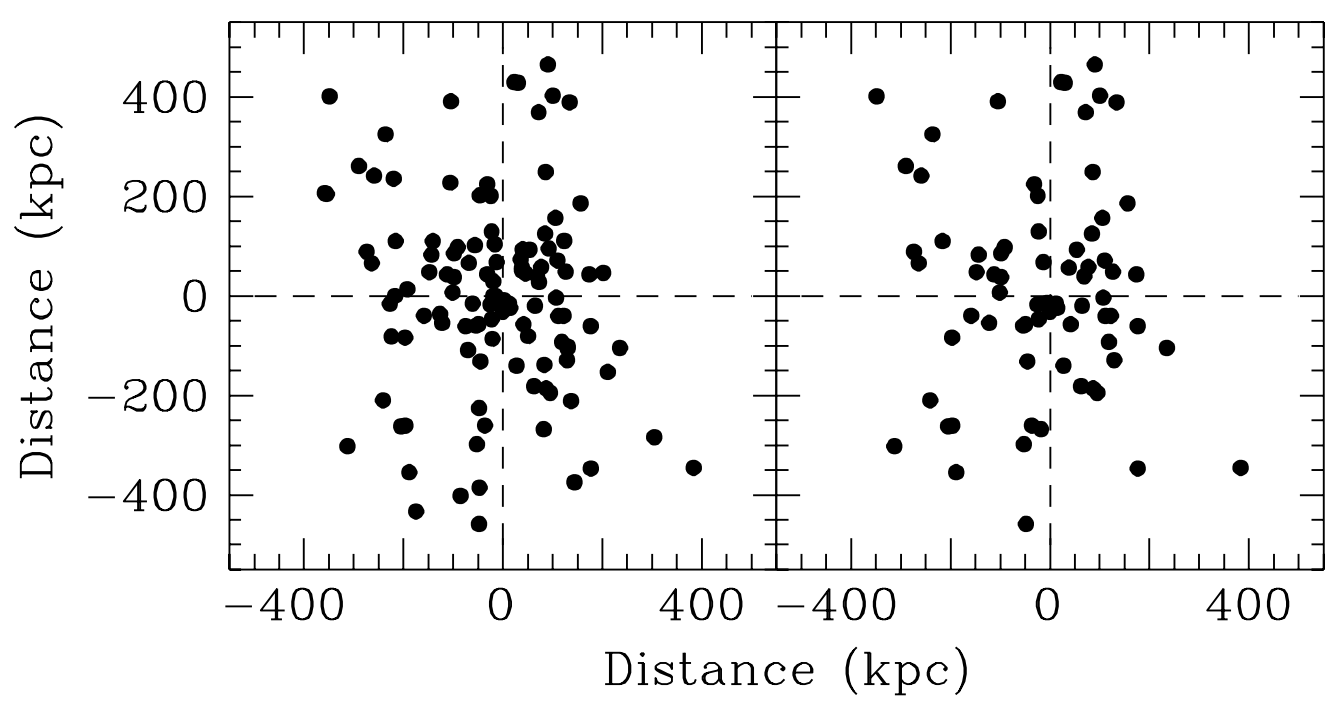


Fig. 2.-

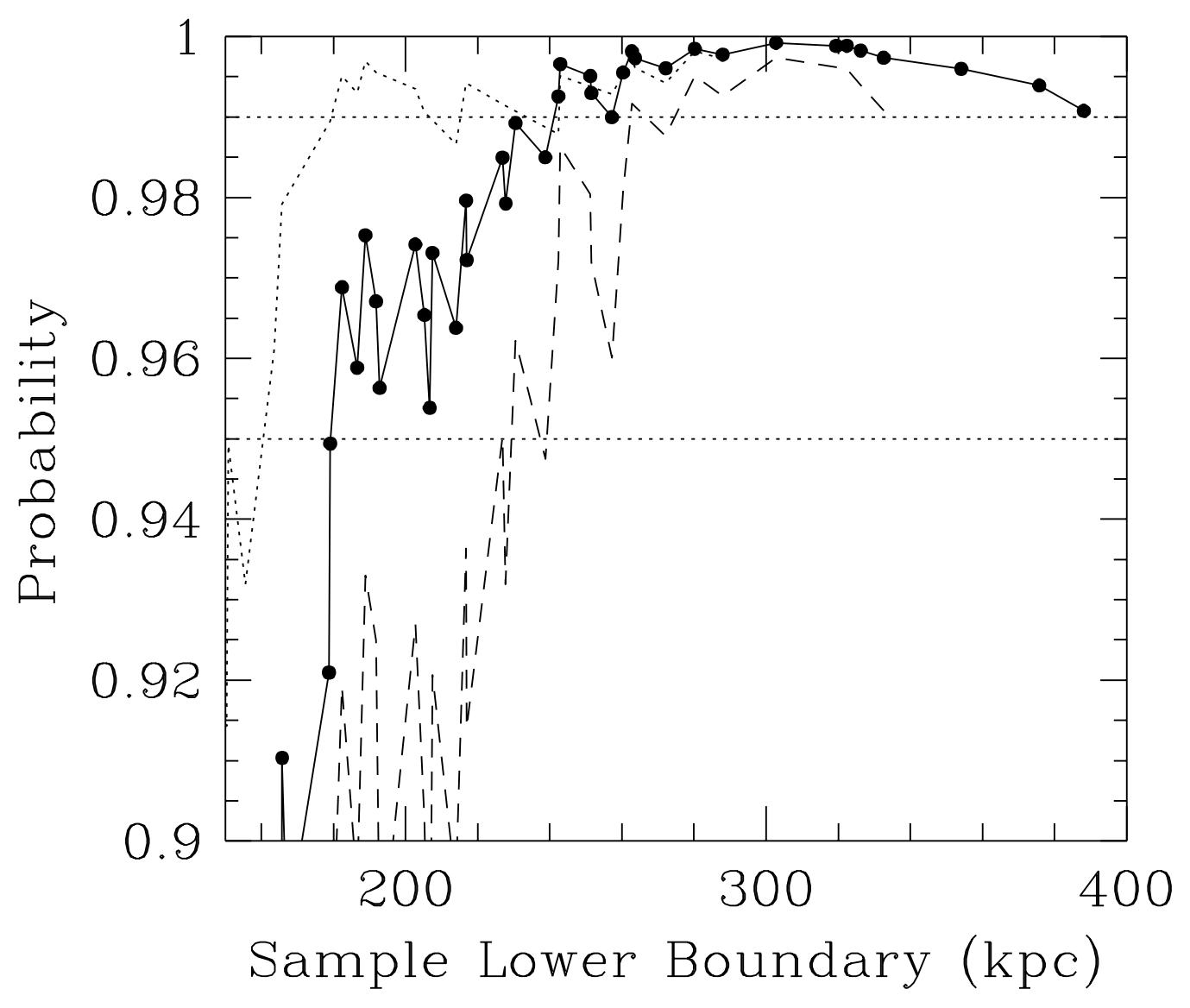


Fig. 3.-

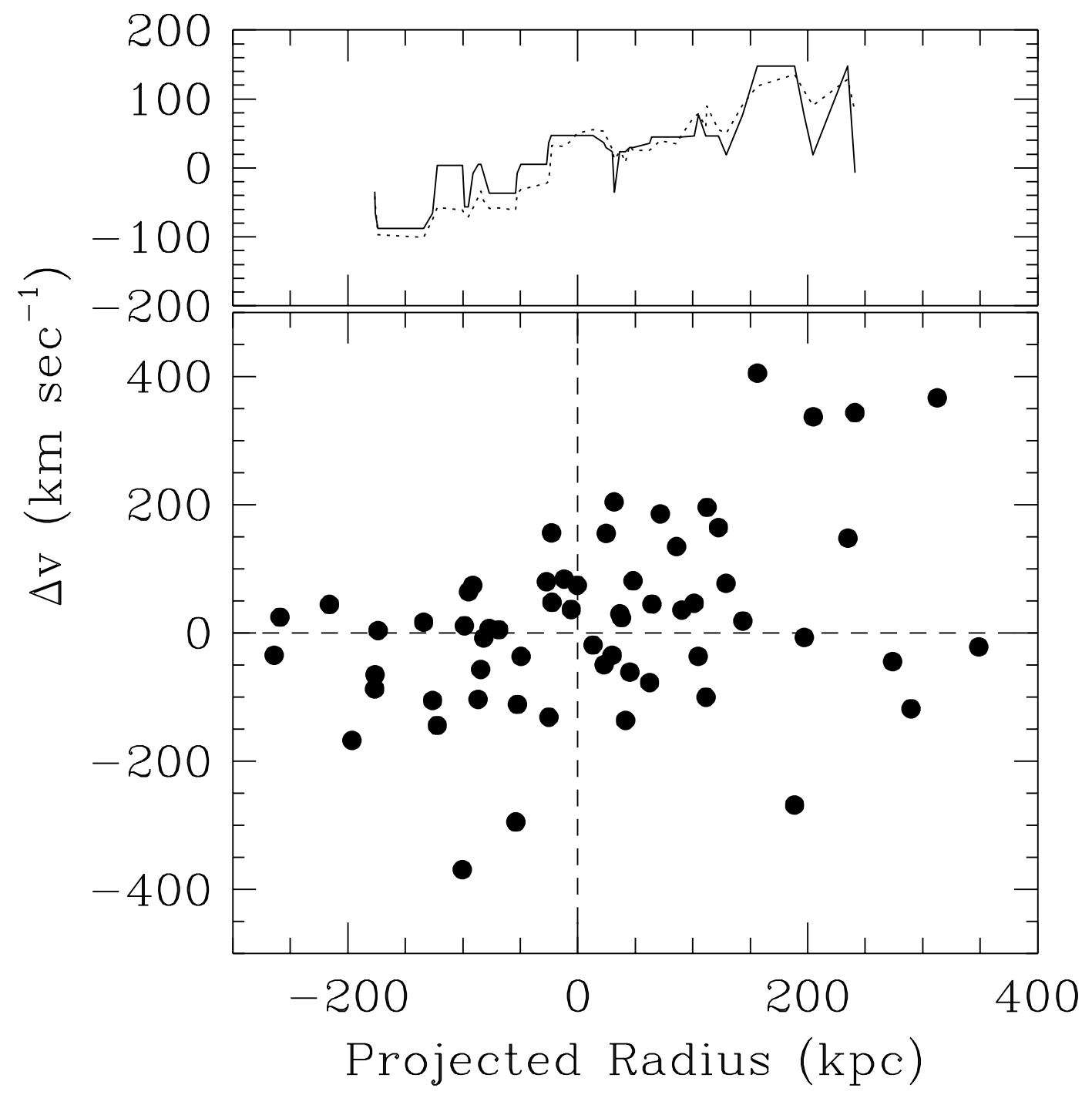

